Radiologe $2021 \cdot 61: 1-2$

https://doi.org/10.1007/s00117-020-00784-1

Angenommen: 27. November 2020

Online publiziert: 8. Januar 2021

(c) Springer Medizin Verlag GmbH, ein Teil von Springer Nature 2020

\section{Fabian Bamberg ${ }^{1} \cdot$ Christian Herold $^{2}$}

${ }^{\prime}$ Klinik für Diagnostische und Interventionelle Radiologie, Universitätsklinikum Freiburg, Medizinische Fakultät, Albert-Ludwigs-Universität Freiburg, Freiburg, Deutschland

${ }^{2}$ Universitätsklinik für Radiologie und Nuklearmedizin, Medizinische Universität Wien, Wien, Österreich

\title{
Wissenschaftskommunikation im Wandel
}

\section{Die neue Rubrik radClub}

Liebe Leserinnen und Leser,

das etablierte System der Wissenschaftskommunikation steht seit den 2000er Jahren mit fortschreitender Digitalisierung seiner wissenschaftlichen Inhalte inmitten der größten Transformation seit Jahrhunderten. Ausgehend von individuellen Briefwechseln zwischen Wissenschaftlern und Ärzten in der Antike, stellten die ersten wissenschaftlichen Zeitschriften Mitte des 17. Jahrhunderts einen radikalen Umbruch dar, so scheint dies von uns gelebte Format auch zunehmend in einem Veränderungsprozess. Dienten in den 90er Jahren noch ausschließlich gedruckte Bücher und Zeitschriften in den Praxen und Kliniken als wichtige Referenz zum Austausch und fachlichen Weiterentwicklung, ergänzen die Verlage zunehmend onlinebasierte Angebote, die über rein digitale Publikationen und Archive hinausgehen [1]. Obgleich diese Entwicklung traditionelle Verlage vor eine besondere Herausforderung stellt, ergeben sich durch die onlinebasierte Ergänzung von digitalen Inhalten ungeahnte Möglichkeiten, die insbesondere in einem weitgehend digitalisierten Fach wie der Radiologie neue Dimensionen ermöglichen.

Damit einher geht auch eine Weiterentwicklung der Wissenschaftsformate und -inhalte, wobei auch hier eine Diversifizierung $\mathrm{zu}$ beobachten ist [2]. Neben der klassischen wissenschaftlichen Publikation im Fachjournal haben sich Wissenschaftsrubriken in der Presse, Wissenschaftsblogs, Social Network Sites oder Twitter etabliert. Insgesamt werden Informationsinhalte entsprechend prägnanter, übersichtlicher und auch kurzfristiger verfügbar. Um dieser Entwicklung auch in Der Radiologe Rechnung zu tragen und radiologisch relevante Inhalte neu zu beleuchten, haben wir eine neue Rubrik entwickelt: rad$C l u b$ wird alle zwei Monate erscheinen und das gewohnte Format in besonderer Weise ergänzen.

Inhaltlich werden wir uns dabei insbesondere mit Themen beschäftigen, welche aus Sicht von Nachwuchsradiologen und -radiologinnen Relevanz für die praktische radiologische Tätigkeit haben. Dies reicht von gelebter Fehlerkultur bis zur praktischen Anleitung beispielsweise für den Einsatz künstlicher Intelligenz. Neu dabei ist, dass für die Artikel im Economist-Style maximal zwei bis drei Druckseiten und nur wenig Literaturstellen zur Verfügung stehen, sodass Inhalte schnell und präzise - auch auf allen mobilen Endgeräten - erfasst werden können. Genutzt wird hierbei zusätzlich der Verweis auf Online-Angebote, auch außerhalb von Der Radiologe, um bei tiefergehendem Interesse ergänzende Informationen, Quelltexte oder Applikationen zur Verfügung zu stellen. Ein besonderes Beispiel ist der erste Artikel innerhalb dieser Rubrik, der von Dr. Molwitz und Kollegen aus Hamburg beigetragen wird. Hierbei geht es um digitalisierte Möglichkeiten der radiologischen Lehre, welche in Zeiten der aktuellen Pandemiesituation einen signifikanten Schub erlebt und die universitäre Lehre vor große Herausforderungen stellt. Neben der studentischen Lehre wird hierbei auch 
innerhalb der radiologischen Weiterbildung die Chance genutzt, neue digitale Lernformate $\mathrm{zu}$ erschaffen und $\mathrm{zu}$ etablieren.

Wir wünschen Ihnen, liebe Leserinnen und Leser, viele neue Erkenntnisse mit radClub und freuen uns, dass diese neue Rubrik auch in Der Radiologe die Wissenschaftstransformation begleitet und weiter vorantreibt.

Ihre

Fabian Bamberg

Christian Herold

\section{Korrespondenzadresse}

Prof. Fabian Bamberg
Klinik für Diagnostische und
Interventionelle Radiologie,
Universitätsklinikum Freiburg,
Medizinische Fakultät, Albert-
Ludwigs-Universität Freiburg
Hugstetter Straße 55,
79106 Freiburg, Deutschland
fabian.bamberg@
uniklinik-freiburg.de

Interessenkonflikt. F. Bamberg und C. Herold geben an, dass kein Interessenkonflikt besteht.

\section{Literatur}

1. Schäfer MS, Kristiansen S, Bonfadelli H (Hrsg) (2015) Wissenschaftskommunikation im Wandel. Halem, Köln

2. Schäfer MS (2014) Vom Elfenbeinturm in die Gesellschaft: Wissenschaftskommunikation im Wandel. Universität Zürich, Zürich (https://nbnresolving.org/urn:nbn:de:0168-ssoar-389155)

\section{Lesen Sie Ihre Zeitschrift online auf SpringerMedizin.de}

SpringerMedizin.de bietet Ihnen Zugang zu allen elektronisch verfügbaren Ausgaben und dem CME-Angebot Ihrer Zeitschrift - unabhängig davon, seit wann Sie die Zeitschrift abonniert haben.

So einfach erhalten Sie Zugang zum Online-Archiv:

- Registrieren Sie sich einmalig auf www.springermedizin.de/register

Geben Sie dabei Ihre Einheitliche Fortbildungsnummer (EFN) an.

- Ihr Benutzername entspricht Ihrer E-Mail-Adresse, Ihr Passwort können Sie frei wählen und später jederzeit unter "Mein Profil" ändern.

- Falls Sie bereits ein (Print-) Abonnement bei uns haben, geben Sie bei der Registrierung die Lieferadresse Ihrer Zeitschrift an. Damit wird Ihr Abo-Zugang auf springermedizin.de freigeschaltet.

Sind Sie bereits bei SpringerMedizin.de registriert?

Dann wird Ihr Zeitschriftenabonnement automatisch Ihrem Online-Nutzerkonto

hinzugefügt. Sollten die Angaben Ihres Online-Accounts nicht eindeutig mit den Angaben Ihres Zeitschriften-Abonnements übereinstimmen, kann die Zuordnung nicht sicher erfolgen. In diesem Fall und bei allen anderen Fragen zum Online-Zugang kontaktieren Sie bitte unseren Kundenservice unter: Kundenservice@springermedizin.de

Telefonisch erreichen Sie die Hotline montags bis freitags von 9.00 bis $17.00 \mathrm{Uhr}$ kostenfrei unter 0800-77 80777 sowie gebührenpflichtig aus dem Ausland unter +4930884293600. 